\title{
Sciendo
}

RURAL SUSTAINABILITY RESEARCH 44(339), 2020

ISSN - 2256-0939

(c) Latvia University of Life Sciences and Technologies, all rights reserved http://www.llu.lv/en/

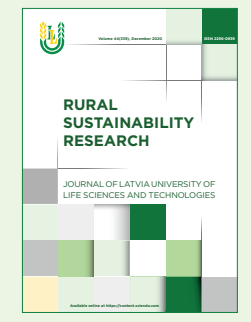

Received: 5 October 2020

Revised: 2 November 2020

Accepted: 9 November 2020

\section{Theoretical Studies of the Vibration Process of the Dryer for Waste of Food}

\author{
Volodymyr Bulgakov ${ }^{1}$, Ivan Sevostianov ${ }^{2}$, Gryhoriy Kaletnik ${ }^{2}$, \\ Ihor Babyn ${ }^{2}$, *Semjons Ivanovs ${ }^{3}$, Ivan Holovach ${ }^{1}$, Yevhen Ihnatiev ${ }^{4}$ \\ ${ }^{1}$ National University of Life and Environmental Sciences of Ukraine, \\ ${ }^{2}$ Vinnytsia National Agrarian University of Ukraine, \\ ${ }^{3}$ Latvia University of Life Sciences and Technologies, \\ ${ }^{4}$ Dmytro Motornyi Tavria State Agrotechnological University
}

\begin{abstract}
An urgent problem is drying and processing of the wet dispersed waste, obtained in the production of food products, which can then be efficiently used as a fertiliser, for feeding livestock or as biofuel. A new design of a vibrating fluidised bed dryer has been developed, which, with low energy consumption, provides a pre-set productivity and the required final moisture content. The process of vertical oscillations of the body of a vibration dryer, together with the food waste contained in it, is analysed analytically, the necessary equivalent scheme is built, on the basis of which differential equations of the vertical oscillations of the body are compiled, their analytical solutions are obtained, and a numerical calculation is performed on a PC using the developed program. Rational parameters of the vibration dryer, providing vibroboiling of the mass of the food waste, have been determined: the body mass $\mathrm{m}=250 \ldots 510 \mathrm{~kg}$; the debalance mass $\mathrm{m}_{\mathrm{d}}=10 \ldots 15 \mathrm{~kg}$; the number of revolutions of the debalance electric motor $\mathrm{n}_{\mathrm{d}}=1950 \ldots 2650 \mathrm{rpm} \cdot \mathrm{min}^{-1}$; maximum stiffness of the support springs $\mathrm{C}_{\mathrm{p}}=8 \cdot 10^{5} \mathrm{~N} \cdot \mathrm{m}^{-1}$; the diameter of the centre of mass of the debalances $\mathrm{d}_{\mathrm{d}}=0.01 \mathrm{~m}$. In addition, as a result of the thermophysical theoretical and experimental studies of the vibration drying process, the following optimal design and technological parameters of the vibration dryer were obtained: the heat transfer area $S_{t . p . n}=4.15 \mathrm{~m}^{2}$; the radius of the heating pipe $r_{t}=0.1 \mathrm{~m}$; the length of the heating pipe $l_{t}=3 \mathrm{~m}$; the number of heating pipes $n_{t}=50$; the heat transfer coefficient $K_{p}=2500$; the final temperature of the dried waste $\mathrm{t}_{\mathrm{o} 2}=100^{\circ} \mathrm{C}$.
\end{abstract}

Keywords: waste, dehydration, vibrations, vibroboiling, model.

\section{Introduction}

In industrial production of the food products, waste is always inevitable (for example, alcohol stillage, brewer's grains, beet pulp, coffee and barley sludge, etc.), which in most cases are not used but accumulate in special containers and, after considerable aging, can be buried in the soil. Application of this food waste after further substantial processing into feed additives or biological fertilisers is still significantly limited due to considerable energy and operating costs (Sevostianov, 2020; Jakovlev et al., 1999).

In addition, an urgent problem and one of the main technological processes of recycling waste, resulting from the production of food products, is their dehydration using various technological equipment (Lehmann et al., 2019; Wang, Zhang \& Deng, 2011; Anytipov, Kretov \& Ostrikov, 2001; Sevostianov, Poliscuk, \& Slabky, 2015, Iskovich-Lotocky, Obertuh, \& Sevostianov, 2006). One of the most widespread and efficient types of dryers for most agricultural materials, including waste, are fluidised and vibroboiling bed devices which provide, in contrast to the drum, tunnel, shaft, belt and roller dryers, a continuous and more efficient working process, achievement of specified values of the final moisture content of the processed material (Yogendrasasidhar

\footnotetext{
* Corresponding Author's email: semjons@apollo.lv
} 
\& Setty Pydi, 2019). Besides, to the devices of the fluidised and vibroboiling bed apparatus belong the spraying, convective, vibrating, spiral, cyclonic, aerial fountain dryers, as well as pneumatic pipe-dryers (Ovchinnikov \& Natareev; 2009; Sazhin, 1984).

In some studies (Ostrikov, Krasovicki, \& Petrov, 2006), various schemes of spray dryers are given: with a central swirling coolant supply (direct-flow); with a central heating agent supply and separate gas and product outlet; with uniform distribution of gases over the cross section through the gas distribution grid; with a radial (along the periphery) coolant supply and central suction. Such a drying equipment provides a sufficiently high productivity of the working process, yet it is characterised by significant energy consumption; in addition, the disadvantages are frequent clogging of the pneumatic nozzles of dryers and their rapid abrasive wear.

During the operation of the vibrating dryers, due to the oscillations of the working chamber, the material in it goes into a state of fluidisation or vibroboiling (Anytipov, Kretov \& Ostrikov, 2001), with mutual separation of particles, their intensive mixing, effective penetration between the coolant particles and the moisture entrainment. This type of the equipment considered, in our opinion, is the most perspective as it provides specified indicators of the final humidity with minimal energy and time consumption. However, as the disadvantage of the above equipment one can consider sufficient complexity and material consumption of the structure. The spray dryer discussed in (Handayani, Utomo \& Yulianto, 2017; Anytipov, Kretov \& Ostrikov, 2001), with mixed flows of the processed material and coolant is distinguished by significant structural complexity, material consumption and large dimensions. It should be noted that auxiliary equipment for the preparation of the coolant, its purification from particles of the processed material, and the separation of the worked out coolant into liquid and gaseous phases is also used in the other devices analysed above. On the whole, for efficient application of the known equipment for drying agricultural products in technological complexes of the waste disposal, obtained in the production of food products, this equipment requires further improvement in the direction of reducing the energy intensity of the working process, reducing the size and increasing the reliability of operation.

The purpose of this study is to increase the efficiency of the dehydration process of the waste obtained in the production of food products through the development of a new design of a vibrating fluidised bed dryer, and theoretical substantiation of the design, kinematic, dynamic and technological parameters.

\section{Materials and Methods}

In carrying out this investigation there were used methods of the designing processes and equipment used in food production, modelling, theoretical mechanics, hydraulics, as well as the method of programming and abandoning programs and conducting numerical calculations on a PC (Dreizler \& Ludde, 2010; Bulgakov et al., 2017).

Figure 1 presents the technological scheme of the developed vibration dryer for processing waste, generated in the production of food products. The technological process of waste drying is carried out in such a way that the waste is fed into the dryer immediately after its preliminary four-stage mechanical dehydration, as a result of which its moisture content reaches no more than $20 \ldots .25 \%$ (Sevostianov \& Lucik, 2017). In addition, the waste is filled into the housing 3 of the vibration dryer, which is provided by the screw feeding conveyor 1 with the shutter 6 open and the shutter 12 closed.

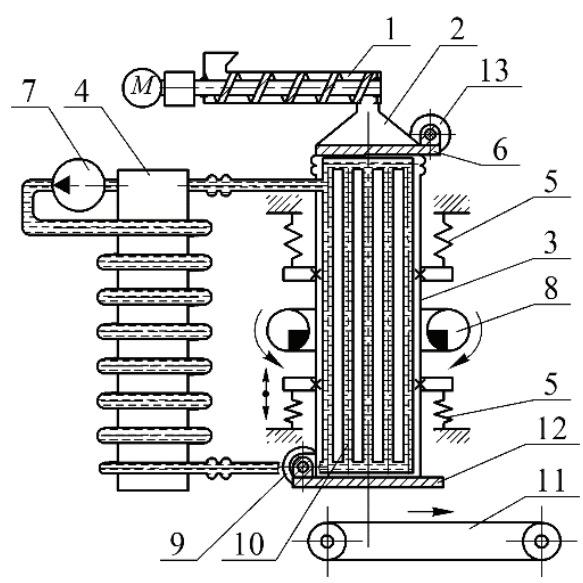

Figure 1. Technological scheme of a vibration dryer for processing waste, obtained in the production of food: the screw feeding conveyor $1 ; 2$ - the conical nozzle; 3 - the housing; 4 - the heat exchanger; 5 springs; 6, 12 - the upper and the lower shutters; 7 the pump of the heat exchanger; 8 - the debalancing vibration exciter; 9,13 - the electric motors; 10 -the

heating pipes; 11 - the outgoing belt conveyor.

The process of loading the food waste into a vibratory dryer continues until housing 3 is filled, after which the screw feeding conveyor 1 is turned off and the shutter 6 is closed. The heating pipes 10 form a rigid structure connected through corrugated rubber pipes to the heat exchanger 4 . The circulation of the coolant through pipes 10 and the coil is provided by pump 7; thus the preparation of the coolant for drying waste is carried out practically without energy consumption. Thermal energy from pipes 10 is transferred to the waste portion at intervals between 
them, ensuring efficient removal of the liquid phase both in the peripheral and in the middle layers of the portion. In addition, to intensify the drying process, housing 3 with the waste is set in vertical oscillatory motion using debalanced vibration exciters 8 rotating at the same speed but in opposite directions. Springs 5 on which the body is suspended hold it in a position, providing the necessary mobility relative to the vertical axis of symmetry of housing 3 , as well as efficient braking at the end of displacements to the boundary upper and boundary lower positions. The mobility of housing 3 relative to the conical nozzle 2 and the mobility of pipes 10 relative to the heat exchanger 4 is ensured by means of corrugated rubber pipes.

Due to the intense vibrational movements of the housing 3 the particles of the portions of waste inside the housing 3 are brought into a state of vibroboiling. This contributes to a more uniform and intensive distribution of heat between the waste particles in the passage sections of the housing 3, which means more efficient and less energy-intensive removal of the liquid phase from them. The latter comes out through small holes in the walls of the housing 3, closed from the inside by a filter mesh. When the predetermined final moisture content of the waste portion in the housing 3 is reached, the electric motor 9 is turned on, which turns and opens the shutter 12. The portion of the dewatered waste is poured onto the outgoing belt 11 , after which the shutter 12 closes again, then the shutter 6 opens and the auger feed conveyor 1 is turned on. The housing 3 is filled with a new portion of waste and the next drying cycle begins. Depending on the type of waste, its initial moisture content and the temperature of the coolant in the pipes, in some cases a continuous mode of waste passage through the housing 3 can be ensured, provided that their pre-set final moisture content is reached. The advantages of the proposed vibratory dryer in contrast to the other types of the above-discussed equipment for drying in a fluidised and vibroboiling bed are the minimum energy consumption for the drying process itself due to the use of heat from the main production, efficient heat transfer to the material particles throughout the section, simplicity and compactness of the design, versatility of application, absence of a possibility of clogging of the sections of the housing, etc. In order to determine the main optimal parameters and evaluate the efficiency of the dryer, we will conduct theoretical studies of the new design of the vibration dryer, developed by us, and establish their relationship with the operating and design parameters of the equipment under study, as well as with the physical and mechanical characteristics of the processed waste.

It is known that, to ensure the transition of particles of a portion of the processed waste located in the body of a vibration dryer to a state of vibrating boiling, the following condition must be met:

$$
\ddot{x}=(2 \ldots 3) g \text {, }
$$

where $\ddot{x}$ - acceleration of the oscillatory movement of the body of the vibration dryer;

$g-$ acceleration of gravity.

Let us provide acceleration of the oscillatory motion of the vibration dryer body indicated in (1), based on the construction of a mathematical model of its oscillations in the longitudinal-vertical plane. Besides it is necessary to draw up differential equations of vertical translational oscillations of the body of the vibration dryer, suspended on springs, during the operation of its debalanced vibration exciters, in order to obtain the dependence of the indicated acceleration upon the design and kinematic parameters of the considered vibration dryer. To build a mathematical model, we will develop an equivalent scheme and make some assumptions. We neglect the elastic forces, created by the corrugated pipes connecting the body of the vibrating dryer with the conical nozzle, and the heating pipes with the coil. The disbalances, created by the electric motor 9 of the damper 6 and the underwater pipes for the coolant, are compensated by counterweights. In addition to it, taking into account the fact that the debalances of the vibration exciter, having the same geometric and dynamic parameters, rotate in opposite directions; the vibrations of the vibration dryer body in the horizontal plane can be ignored. This equivalent scheme is shown in Figure 2. In this case we consider the body of the vibration dryer in the form of a separate body, which is mounted on a frame on several springs and can move in a vertical plane up and down during the operation of the vibration exciters rigidly connected to it. Figure 2 shows the position of the body of the vibrating dryer at an arbitrary moment in time $t$ when it performs a translational vertical vibration. In addition, the equivalent scheme shows a case where the body of the vibrating dryer is located below its static equilibrium position. Let us show on the equivalent scheme the coordinate system $O x$ with the origin at the point $\mathrm{O}$. The vertical axis $x$ coincides with the longitudinal axis of symmetry of the vibration dryer body and along which its body performs translational vertical oscillations.

Besides, the position of the vibration dryer body at an arbitrary moment of time $t$ is characterised by coordinate $x$ of the body deviation from the equilibrium position. In Figure 2 just a case is shown when the body of the vibration dryer is below its equilibrium position by exactly $x$. In this case $-x_{\max } \leq x \leq 0$, where $x_{\max }$ is the vibration amplitude 


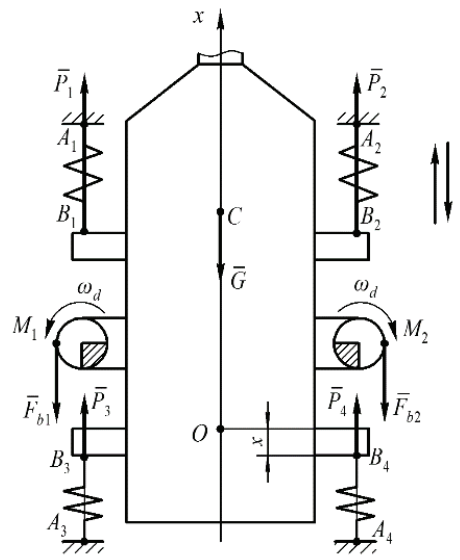

Figure 2. Equivalent scheme of the oscillatory process of the vibration dryer.

of the vibration dryer body. In the case when the body of the vibrating dryer is above its equilibrium position, the following condition will take place: $0 \leq x \leq x_{\max }$.

When the body is moving up, the springs $A_{1} B_{1}$, $A_{2} B_{2}$ are compressed, and the springs $A_{3} B_{3}, A_{4} B_{4}$ are stretched out $\left(A_{1}, A_{2}, A_{3}, A_{4}\right.$ and $B_{1}, B_{2}, B_{3}, B_{4}$ are the points of attachment of the springs to the frame and the body of the vibration dryer, respectively). When the vibration dryer body is moving down, springs $A_{1} B_{1}, A_{2} B_{2}$ are stretched out, and springs $A_{3} B_{3}, A_{4} B_{4}$ are compressed. The maximum value of deformation of the springs is equal to two amplitudes of vibration of the body, that is $2 x_{\max }$. The stiffnesses of the springs used are denoted by $C_{p i}(i=1,4)$. Thus the elastic forces, generated by the springs, are the restoring forces in the considered oscillatory process. Oscillations of the vibration dryer body are carried out under the influence of two debalanced vibration exciters that generate centrifugal inertial forces, the vertical components of which are the disturbing forces of these vibrations.

The following forces are shown in the equivalent scheme:

$\bar{F}_{b 1}, \quad \bar{F}_{b 2}$ - disturbing forces of the forced translational vibrations of the vibration dryer body (the vertical components of centrifugal forces of inertia of rotation of the debalanced vibration exciters, applied at points $M_{1}$ and $M_{2}$, respectively);

$\bar{P}_{1}, \bar{P}_{2}, \bar{P}_{3}, \bar{P}_{4}$ - elastic forces of longitudinal deformation of springs $A_{1} B_{1}, A_{2} B_{2}, A_{3} B_{3}, A_{4} B_{4}$ applied at points $B_{1}, B_{2}, B_{3}, B_{4}$, respectively;

$\bar{G}$ - the force of the weight of the vibration dryer body together with a portion of the processed waste, applied in the centre of mass of the vibration dryer body (point $C$ ).

In Figure 2, there is also shown the vertical movement $x$ of the vibration dryer body from its equilibrium position downward at an arbitrary moment in time $t$.
Let us further determine the values of all the forces shown in the equivalent scheme (Figure 2).

It is obvious that forces $F_{b i}(i=1,2)$ as vertical components of the centrifugal forces of inertia of the rotation of the debalances will be equal:

$$
\bar{F}_{b i}=m_{d} \cdot r_{d} \cdot \omega_{d}^{2} \cdot k_{d} \cdot \sin \left(\omega_{d} \cdot t\right),(i=1,2),
$$

where $m_{d}$ - the debalance mass; $r_{d}$ - the radius of the debalance circle of rotation; $\omega_{d}-$ the angular speed of rotation of the vibration exciter shaft; $k_{d}$ - the number of debalances of the vibration exciter; $t$ - the current time.

In case $n_{d}$ debalance revolutions per minute are set, as well as diameter $d_{d}$ of the debalance rotation circle, then, considering that:

$$
\omega_{d}=\frac{\pi \cdot n_{d}}{30}
$$

from formula (2), after substitution of dependence (3), we obtain the following expression for determination of the magnitude of the forces $\bar{F}_{b i}$ :

$F_{b i}=\frac{\pi^{2} \cdot m_{d} \cdot d_{d} \cdot n_{d}^{2} \cdot k_{d}}{1800} \cdot \sin \left(\frac{\pi \cdot n_{d}}{30} \cdot t\right),(i=1,2)$.

The gravity force $G$ of the vibration dryer body, together with the technological material, will be equal to:

$$
G=m \cdot g
$$

where $m$ - the total mass of the vibration dryer body of the recyclable waste and all other vibrating structural elements; $g$ - acceleration of gravity.

To determine the elastic forces $\bar{P}_{i}, i=\overline{1,4}$, the longitudinal deformation of the springs at an arbitrary moment of time $t$, the following should be remarked. We will assume that in a state of static equilibrium, the 
lower springs $A_{3} B_{3}$ and $A_{4} B_{4}$ are compressed by value $l_{s t}$, and the upper springs $A_{1} B_{1}$ and $A_{2} B_{2}$ are stretched out by value $l_{s t}$. In addition, the elastic forces of static deformation of all four springs will be directed upward (Figure 2).

Obviously the elastic force of the static deformation of each spring will be equal to:

$$
P_{i}=C_{p} l_{s t}=C_{p} \frac{G}{4 \cdot C_{p}}=\frac{1}{4} m g, \quad i=\overline{1,4},
$$

where $C_{p}$ - the stiffness of the spring.

There is also possible a variant of suspension of the body when the upper springs are attached to the frame at a height at which they are free in a static equilibrium position; then the gravity force $G$ of the body will be balanced by the forces of longitudinal compression of only the two lower springs. In this case:

$$
P_{1}=P_{2}=0, \quad P_{3}=P_{4}=\frac{1}{2} m g \text {. }
$$

In the first variant the elastic forces of the springs change their direction during one period of vibration of the vibration dryer body; therefore, it is necessary to consider four stages of moving the vibration dryer body during the this period.

Stage I - moving the body of the vibration dryer from equilibrium into the uppermost position.

Stage II - moving the body from the uppermost to the equilibrium position.

Stage III - moving the body from equilibrium to the lowest position.

Stage IV - moving the body of the vibration dryer from the lowest to the equilibrium position.

Let us discuss the behaviour of the elastic forces of the longitudinal deformation of the springs at stage I. In this case, at the beginning of the movement of the vibration dryer body up from the position of static equilibrium, its gravity force will be balanced by forces (6) of the static deformation of the springs, as shown in Figure 2. However, when the body is moved upward at a distance $x=l_{s t}$ (we start the fixation of the movement $x$ from the static equilibrium position, point), all four springs will become free (the length of the upper springs $A_{1} B_{1}$ and $A_{2} B_{2}$ will decrease by value $l_{s t}$, and the length of the lower springs $A_{3} B_{3}$ and $A_{4} B_{4}$ will increase by the same value; that is, all the springs will acquire their design length when unloaded). Thus, at $x=l_{s t}, P_{i}=0, i=1.4$. The case of moving the body upward at a distance $x=l_{s t}$ from the position of static equilibrium has a one-time character, only at the beginning of the start-up of the dryer. In this case

$$
P_{i}=C_{p}\left(l_{s t}-x\right), \quad i=\overline{1,4}, \quad 0 \leq x \leq l_{s t},
$$

In addition, all these forces will be directed upward. Although it should be noted that the balance of the force of the body weight by the forces of elastic deformation of the springs at the beginning of the start-up of the dryer has a positive value. The debalanced exciters at the moment of starting the dryer have to overcome only the force of inertia of the vibration dryer body, and not its weight. Thus, the equation of the movement of the body at $x>l_{s t}$ is of greatest interest. Obviously, with further movement of the body upward $\left(x>l_{s t}\right)$, deformation of the springs will be equal to $l_{p}=x-l_{s t}$. Indeed, at $x=l_{s t}, l_{p}=0$, as was already noted above. Only for the upper springs $A_{i} B_{i}, i=\overline{1.2}$, this will be the longitudinal compression deformation, but for the lower springs $A_{i} B_{i}, i=\overline{3.4}-$ the longitudinal stretching. Therefore, at the discussed stage of movement of the vibration dryer body of the elastic forces $\bar{P}_{i}, i=\overline{1.4}$, all the springs will be directed downward, that is, against the direction of movement of the body. At this stage the indicated elastic forces will be equal:

$$
P_{i}=C_{p}\left(x-l_{s t}\right), \quad i=\overline{1,4}, \quad l_{s t} \leq x \leq x_{\max },
$$

In addition, in the extreme upper position of the body the elastic forces $\bar{P}_{i}, i=1.4$ reach their maximum value $P_{i \max }$ and will be equal to:

$$
P_{i \max }=C_{p}\left(x_{\max }-l_{s t}\right), \quad i=\overline{1,4},
$$

where $x_{\max }$ - maximum upward movement of the body from the static balance position.

Thus, at stage I the elastic deformation forces are within the range from 0 to $P_{i \max }$, that is. $0 \leq P_{i} \leq P_{i \max }, i=\overline{1.4}$. Let us further define expressions for determination of the elastic forces of the springs at stage II. It is obvious that when the body moves from its upper position downward by value $x$, the degree of the longitudinal deformation of the springs becomes equal to $l_{p}=x_{\max }-l_{s t}-x$. Indeed, at $x=0, l_{p}=x_{\text {max }}-l_{s t}$, but at $x=x_{\text {max }}-l_{s t}, l_{p}=0$ (the springs are released). At this stage for the upper springs $A_{i} B_{i}, i=\overline{1.2}$ this will be the longitudinal stretching deformation, but for the lower springs $A_{i} B_{i}, i=\overline{3.4}$ - the longitudinal compression. Therefore, at stage II of the movement of the body of the vibration dryer, forces $\bar{P}_{i}, i=\overline{1.4}$ will be directed downward (the upper springs are still compressed the lower ones, on the contrary, are stretched out). Thus, in this position forces $\bar{P}_{i}, i=\overline{1.4}$ are directed towards the movement of the body. At this stage the indicated elasticity forces of the spring will be equal:

$P_{i}=C_{p}\left(x_{\max }-l_{s t}-x\right), \quad i=\overline{1,4}, 0 \leq x \leq x_{\max }-l_{s t}$, 
Besides, at this stage forces $\bar{P}_{i}, i=\overline{1.4}$ vary from $P_{i \max }$ to 0 , that is, $0 \leq P_{i} \leq P_{i \max }, i=\overline{1.4}$. Let us consider further stage III of the movement of the vibration dryer body. At the initial moment of time of this stage all the springs are free, therefore $\bar{P}_{i}=0, i=\overline{1.4}$. With further downward movement of the body, the upper springs $A_{i} B_{i}, i=\overline{1.2}$ continue stretching, but the lower springs $A_{i} B_{i}, i=\overline{3.4}$ continue contracting. However, the elastic forces $\bar{P}_{i}, i=\overline{1.4}$ of all the springs will already be directed upwards; besides, forces $\bar{P}_{i}, i=\overline{1.2}$ are the forces of longitudinal tensile deformation, but $\bar{P}_{i}, i=\overline{3.4}$ the forces of compression. Consequently, forces $\bar{P}_{i}, i=\overline{1.4}$ will be directed against the movement of the body. In this case the value of the longitudinal deformation of the springs when the body is moving by value $x$ will be equal to $l_{p}=l_{s t}-x$. Indeed,

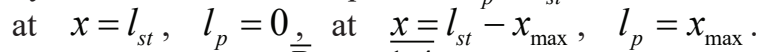
Therefore, forces $\bar{P}_{i}, i=\overline{1.4}$ in this case will be equal to:

$$
P_{i}=C_{p}\left(l_{s t}-x\right), \quad i=\overline{1,4}, l_{s t} \leq x \leq x_{\max } .
$$

Obviously, at stage III the value of forces $\bar{P}_{i}$ varies within a range from 0 to $P_{i \max }$, that is, $\quad 0 \leq P_{i} \leq P_{i \max }, i=\overline{1.4}$, where $P_{i \max }=C_{p}\left(l_{s t}-x_{\max }\right), \quad i=1.4$.

And, finally, we consider stage IV of the movement of the vibration dryer body. It is obvious that by moving the body from its lowest position upward by value $x$, the value of the longitudinal deformation of the springs becomes equal to $l_{p}=l_{s t}-x_{\max }+x$. Indeed, at $x=0, l_{p}=l_{s t}-x_{\max }$, but at $x=x_{\max }-l_{s t}$, $l_{p}=0$, (the springs are free). At the stage considered the upper springs $A_{i} B_{i}, i=\overline{1.2}$ are compressed, but the lower springs $A_{i} B_{i}, i=\overline{3.4}$ are stretched. Consequently, at stage IV the elastic forces of the longitudinal deformation of the springs, when the body moves upward by value $x$, will be:

$$
P_{i}=C_{p}\left(l_{s t}-x_{\max }+x\right), \quad i=\overline{1.4}, 0 \leq x \leq x_{\max }-l_{s t} .
$$

In addition, the forces of elasticity of the springs $\bar{P}_{i}, i=\overline{1.4}$ vary from $P_{i \max }$ to 0 , that is, $0 \leq P_{i} \leq P_{i \max }, i=\overline{1.4}$ where $P_{i \max }=C_{p}\left(l_{s t}-x_{\max }\right)$. Finally, it should be remarked that at stage IV of the movement of the body the elastic forces of all four springs will be directed upwards, that is, in the direction of the movement of the body of the vibration dryer.

Based on the equivalent scheme, developed by us (Figure 2), we will write down a differential equation of the movement of the oscillating body of the vibratory dryer with recyclable waste, using the basic law of dynamics in a vector form:

$$
m \bar{a}=\bar{F}_{b 1}+\bar{F}_{b 2}+\bar{P}_{1}+\bar{P}_{2}+\bar{P}_{3}+\bar{P}_{4}+\bar{G}
$$

where $\bar{a}$ - acceleration of the vibrating body of the vibration dryer.

When compiling differential equations of the movement of the body with waste on the basis of the vector equation (14), we take the direction of the movement of the body at one or another stage of its oscillation cycle as the positive direction of forces. Let us first write down a differential equation of the movement of the vibration dryer body at the stage of starting the machine. According to the equivalent scheme (Figure 2), this differential equation will have the following form:

$$
\begin{aligned}
& m \ddot{x}=\frac{\pi^{2} \cdot m_{d} \cdot d_{d} \cdot n_{d}^{2} \cdot k_{d}}{900} \cdot \sin \left(\frac{\pi \cdot n_{d}}{30} \cdot t\right)+ \\
& +4 C_{p}\left(x-l_{s t}\right)-m g, 0 \leq x \leq l_{s t} .
\end{aligned}
$$

This equation, as mentioned above, takes place only at the stage of starting the machine. When determining the values of forces $(i=\overline{1.4})$, the

Stage I:

$$
\begin{aligned}
& m \ddot{x}=\frac{\pi^{2} \cdot m_{d} \cdot d_{d} \cdot n_{d}^{2} \cdot k_{d}}{900} \cdot \sin \left(\frac{\pi \cdot n_{d}}{30} \cdot t\right)-4 C_{p}\left(x-l_{s t}\right)-m g, \\
& l_{s t} \leq x \leq x_{\max }, t=0 \ldots \frac{15}{n_{d}} .
\end{aligned}
$$

Stage II:

$$
\begin{aligned}
& m \ddot{x}=\frac{\pi^{2} \cdot m_{d} \cdot d_{d} \cdot n_{d}^{2} \cdot k_{d}}{900} \cdot \sin \left(\frac{\pi \cdot n_{d}}{30} \cdot t\right)+4 C_{p}\left(x_{\max }-l_{s t}-x\right)+m g, \\
& 0 \leq x \leq x_{\max }-l_{s t}, t=\frac{15}{n_{d}} \ldots \frac{30}{n_{d}} .
\end{aligned}
$$


Stage III:

$$
\begin{aligned}
& m \ddot{x}=\frac{\pi^{2} \cdot m_{d} \cdot d_{d} \cdot n_{d}^{2} \cdot k_{d}}{900} \cdot \sin \left(\frac{\pi \cdot n_{d}}{30} \cdot t\right)-4 C_{p}\left(l_{s t}-x\right)+m g, \\
& l_{s t} \leq x \leq x_{\max }, \quad t=\frac{30}{n_{d}} \ldots \frac{45}{n_{d}} .
\end{aligned}
$$

Stage IV:

$$
\begin{aligned}
& m \ddot{x}=\frac{\pi^{2} \cdot m_{d} \cdot d_{d} \cdot n_{d}^{2} \cdot k_{d}}{900} \cdot \sin \left(\frac{\pi \cdot n_{d}}{30} \cdot t\right)+4 C_{p}\left(l_{s t}-x_{\text {max }}+x\right)-m g, \\
& 0 \leq x \leq x_{\max }-l_{s t}, \quad t=\frac{45}{n_{d}} \ldots \frac{60}{n_{d}} .
\end{aligned}
$$

oscillation period of the body of the vibration dryer with waste was divided into four stages. Therefore the differential equations of the movement of the body must also be written for each of these stages. Based on the equivalent scheme and vector equation (14), taking into account expressions (4), (5), (9) - (13), the mentioned differential equations in projections onto the Ox axis will have the following form:

For numerical solution and analysis of differential equations (16) - (19) it is necessary to set the design and kinematic parameters of the vibration dryer. First of all, it is necessary to determine the mass of the housing together with the food waste in it, for which it is necessary to determine the mass of the waste itself and the masses of the component parts of the housing, vibrating together with it. Therefore, we will consider and determine the necessary parameters of the waste drying process itself. For this purpose let us consider the initial mass parameters, used in a real enterprise for processing the food waste (Sevostianov \& Lucik, 2017).

Based on the daily mass $\mathrm{T}_{\mathrm{c}}$ of tonnes of waste at an enterprise using a vibratory dryer of this design, we calculate their mass $m_{o}$ after four-stage mechanical dehydration (Sevostianov, 2020) to a moisture content of $U_{k . o}=25 \%$, that is, the mass of waste entering directly into the vibration dryer. In this case, we use the expression: in this case we use the expression:

$$
\begin{aligned}
& m_{o}=m_{c}-m_{c} \cdot \frac{U_{n . o}-U_{k . o}}{100}=m_{c}- \\
& -m_{c} \frac{95-25}{100}=0.3 \cdot m_{c},
\end{aligned}
$$

where $U_{n . o}=95 \%$ - the initial moisture content of the food waste.

The total time $t_{z}$ for loading the waste with mass $m_{o}$ into the described vibration dryer is found taking into account the efficiency of the screw feeder $Q_{s}$ from the following expression (Anytipov, Kretov \& Ostrikov, 2001).

$$
t_{z}=\frac{m_{o}}{Q_{s}}=\frac{m_{o}}{\left[\frac{m_{s} \cdot k_{s}}{4} \cdot\left(D_{s}^{2}-d_{s}^{2}\right)\right] \cdot\left[t_{s}-\frac{b_{1}-b_{2}}{2 \cos \alpha}\right] \cdot n_{s} \cdot \rho_{o . o} \cdot k_{1} \cdot k_{2} \cdot k_{3}},
$$

where $m_{s}, k_{s}, D_{s}, d_{s}, t_{s}, b_{1}, b_{2}, \alpha, n_{s}$ - the number of the screw entries, the number of screws, the largest and the smallest screw diameters, the pitch of the screw, the width of the helical blade in section along the inner and outer radii of the screw, the angle of ascent of the helical line along the average diameter of the screw, the rotation frequency of the feeder auger, respectively; $k_{1}, k_{2}, k_{3}$ - the coefficients of filling the inter-screw space $\left(k_{1}=0.9 \ldots 1.0\right)$, waste compression $\left(k_{2}=0.51 \ldots 0.56\right)$, the supply reduction of the feeder $\left(k_{3}=0.9\right)$, respectively; $\rho_{0.0}-$ density of the waste dehydrated to a moisture content of $25 \%$.

The daily mass $m_{c . o}$ of the waste after drying to the moisture content $U_{c . o}=8 \%$ is calculated as:

$$
\begin{aligned}
& m_{c . o}=m_{o} \frac{U_{k . o}-U_{c . o}}{100}=m_{o} \frac{25-8}{100}= \\
& =0.17 \cdot m_{o}=0.051 \cdot m_{c} .
\end{aligned}
$$

The time $t_{r}$ for unloading the waste with a mass $m_{c . o}$ from the vibration dryer is determined based on the main parameters of the belt conveyor according to the formula:

$$
t_{r}=\frac{m_{c . o}}{\rho_{c . o} \cdot v_{k} \cdot B_{k} \cdot h_{k}},
$$

where $\rho_{c . o}-$ density of the waste dewatered to the moisture content of $8 \% ; v_{k}$ - the speed of the conveyor belt; $B_{k}$ - the working width of the belt; $h_{k}$ - thickness of the layer of the dried waste on the belt - corresponds to the gap between the bottom end of the housing with the shutter open and the surface of the belt.

Next, it is necessary, using an experimental prototype of a vibration dryer, to experimentally set the drying time $t_{s . p}$ for a portion of waste with a mass 
$m_{p . e}$ to the moisture content of $U_{k . c}=8 \%$, with a filled body and closed shutters.

Based on $t_{s . p}$, we find the number $n_{p}$ of the waste portions dehydrated by the installation:

$$
n_{p}=\frac{\left[24 \cdot 3600-\left(t_{z}-t_{r}\right)\right]}{t_{s . p}},
$$

We find mass $m_{p . p}$ of a portion of waste with moisture $U_{k . o}$, which the industrial vibratory dryer must dehydrate in time $t_{s . p}$, by the formula:

$$
m_{p \cdot p}=\frac{m_{o}}{n_{p}}
$$

The required surface area $S_{t . p}$ of the heating pipes of the industrial installation is determined from the ratio:

$$
S_{t . p}=\frac{m_{p \cdot p}}{m_{p . e}} S_{t . e},
$$

where $S_{t . e}-$ is the surface area of the heating pipes of the experimental prototype of the vibration dryer.

The length $l_{t}$ of the heating pipes is set based on the height of the ceiling of the workshop in which it is planned to install the vibration dryer, the height of the screw feeder, the height of the conical nozzle $-h_{n}$ , the size of the gaps $-l_{z v}$ between the upper end of the pipes and the upper end of the dryer body, $-l_{z n}$ between the lower end of the pipes and the end of the body $\left(l_{z v}, l_{z n}\right.$ correspond to similar parameters of the experimental prototype), the working length $l_{z}$ of the shutter in an open position, thickness $h_{k}$ of the layer of the dried waste on the belt and the height of the conveyor.

Then the diameter of the heating pipes $d_{t}$ inside the housing can be calculated as:

$$
d_{t}=\frac{S_{t \cdot p}}{\pi \cdot l_{t} \cdot n_{t}}
$$

where $n_{t}-$ the number of the heating pipes.

The height $h_{k}$ and radius $r_{k}$ of the vibration dryer body is determined by the following formulas:

$$
\begin{aligned}
& h_{k}=l_{t}+l_{z v}+l_{z n} ; \\
& r_{k}=\sqrt{\frac{\left[\frac{\pi d_{t}^{2}}{4} l_{t} n_{t}+\frac{m_{p . p}}{\rho_{o . o}}\right]}{\pi \cdot h_{k}} .}
\end{aligned}
$$

Knowing the external dimensions of the heating pipes and the body of the vibration dryer, from the design considerations we determine their thickness and, taking into account the experience of designing vibration dryers (Ostrikov, Krasivicki, \& Petrov, 2006), select the material for the manufacture of these elements, after which we calculate their masses. Based on radius $r_{k}$ of the vibration dryer body and design considerations, we determine the dimensions and mass $m_{z}$ of the shutter, taking into account the mass of its attachment unit. We also select electric motors and debalanced vibration exciters, and determine their mass. Further, having all the necessary data, we calculate the total mass of the vibration dryer body together with the portion of waste contained in it:

$m=m_{p \cdot p}+m_{t}+m_{k}+m_{z}+n_{v} \cdot m_{v}+m_{e}+m_{t e p}$

where $m_{t}, m_{k}, m_{v}, m_{e}-$ masses of the heating pipes, housing (taking into account the mass of spring attachments), vibration exciter and electric motor, respectively; $m_{\text {tep }}-$ mass of the heat carrier in the total volume of the heating pipes $\left(V_{1}=\frac{\pi \cdot d_{t}^{2}}{4} l_{t} \cdot n_{t}\right.$ taken into account when using a liquid heat carrier for drying); $n_{v}$ - the number of vibration exciters.

We also select springs of certain stiffness that fasten the body to the frame, after which we have the opportunity to solve differential equations (16) (19) by numerical methods on a computer. Based on the results obtained, we check the condition for the transition of particles of the waste portion in the housing during the drying process to the state of vibroboiling according to expression (1). If condition (1) is not met, then, by changing the parameters included in equations (16) - (19), and by repeatedly solving these equations, we achieve the fulfilment of condition (1). In this case we set the frequency $v=35$ $\mathrm{Hz}$ of the body oscillations, which takes place when drying the coffee sludge. This value of frequency $v$ was obtained in the course of experiments with vibro-shock dehydration of the portions of the coffee sludge on an experimental hydraulic impulse stand (Sevostianov, 2020). As experiments have shown, when the indicated frequency is implemented on the workbench, the maxi, mum efficiency of the vibroshock sludge dewatering is ensured with a minimum energy intensity of the working process, reaching the final moisture content of the waste $21 . .22 \%$. It should be considered that with vibration drying of this type of waste, using frequency $v=35 \mathrm{~Hz}$, the efficiency of the working process will also be close to maximum.

Based on the value of $v$, we calculate the required rotational speed of the debalanced vibration exciters $-n_{d}$. Considering that $v=\frac{1}{T_{d}}$, but $T_{d}=\frac{60}{n_{d}}$, we obtain the frequency value $n_{d}$ :

$$
n_{d}=60 \cdot v=60 \cdot 35=2100 \mathrm{rpm} \cdot(\min )^{-1} \text {. }
$$


This value can be ensured by the industrial debalanced vibration exciters of model IV-99.1 (Sevostianov \& Lucik, 2017) with a maximum driving force $F_{d}=5 \mathrm{kN}$ and an adjustable debalance rotation frequency $n_{d}=1500 \ldots 3000 \mathrm{rpm} \cdot(\mathrm{min})^{-1}$.

On the basis of the design calculations according to expression (29) and analysis of the production analogs of machines, as well as experimental investigations, we regard the mass of the vibration dryer body together with the waste to be equal to $510 \mathrm{~kg}$. Thus we obtain all the necessary data for solving equations (16) - (19). However, it is enough for us to solve only the first of these four equations, namely, equation (16), which describes the upward movement of the body as a state of the highest loading under the action of external forces. The parameters obtained as a result of solving this equation will all the more provide the necessary operating mode in case of moving the body down. Since equation (16) is a second-order linear differential equation, its solution can be obtained in quadratures, that is, it is possible to obtain its analytical solution. Taking into account expression (6), we obtain that $m g=4 C_{p} \cdot l_{s t}$ (the weight of the body is balanced by the elastic forces of the four springs).

Taking into account this remark, after some transformations, equation (16) assumes the following form:

$\ddot{x}=\frac{\pi^{2} \cdot m_{d} \cdot d_{d} \cdot n_{d}^{2} \cdot k_{d}}{900 \cdot m} \cdot \sin \left(\frac{\pi \cdot n_{d}}{30} \cdot t\right)-\frac{4 C_{p}}{m} \cdot x$.

Further we denote:

$$
\frac{\pi^{2} \cdot m_{d} \cdot d_{d} \cdot n_{d}^{2} \cdot k_{d}}{900 \cdot m}=H \text {. }
$$

Taking into account expressions (3) and (32), equation (31) will be written in the following form:

$$
\ddot{x}=H \cdot \sin \left(\omega_{d} \cdot t\right)-\frac{4 C_{p}}{m} \cdot x,
$$

or

$$
\ddot{x}+\frac{4 C_{p}}{m} \cdot x=H \cdot \sin \left(\omega_{d} \cdot t\right) .
$$

Let's introduce other notation:

$$
\frac{4 C_{p}}{m}=k^{2}, \quad \text { or } \quad 2 \sqrt{\frac{C_{p}}{m}}=k .
$$

Taking into account (35), equation (34) will have the following form:

$$
\ddot{x}+k^{2} \cdot x=H \cdot \sin \left(\omega_{d} \cdot t\right) .
$$

By solving this equation analytically, we obtain:

$$
x=l_{s t} \cdot \cos (k \cdot t)+\frac{H}{k^{2}-\omega_{d}^{2}} \cdot \sin \left(\omega_{d} \cdot t\right) .
$$

This solution was found under the following initial conditions:

$$
\begin{aligned}
& \text { At } t=0: \\
& x=x_{0}=l_{s t}, \dot{x}=\dot{x}_{0}=0 .
\end{aligned}
$$

Besides, in expression (37):

$l_{s t}$ - the amplitude of free (natural) oscillation of the vibration dryer body;

$k=2 \sqrt{\frac{C_{p}}{m}}-$ frequency of the natural oscillations themselves;

$\frac{H}{k^{2}-\omega_{d}^{2}}-$ amplitude of forced oscillations of the vibration dryer body.

Expression (37) describes the law of oscillations of the vibration dryer body in time.

Variation in the speed of the movement of the vibration dryer body in time $t$ will be determined by the following expression:

$$
\dot{x}=-k \cdot l_{s t} \cdot \sin (k \cdot t)+\frac{H \cdot \omega_{d}}{k^{2}-\omega_{d}^{2}} \cdot \cos \left(\omega_{d} \cdot t\right) .
$$

\section{Results and Discussion}

By differentiating expression (39) with respect to time $t$, we obtain the law of variation in the acceleration of the vibration dryer body in time $t$ :

$$
\ddot{x}=-k^{2} \cdot l_{s t} \cdot \cos (k \cdot t)-\frac{H \cdot \omega_{d}^{2}}{k^{2}-\omega_{d}^{2}} \cdot \sin \left(\omega_{d} \cdot t\right) .
$$

Substituting various values of the parameters, included in (40), we obtain the necessary acceleration values $\ddot{x}$ of the vibrodryer body, providing vibroboiling of the mass of waste located at that time in the middle of the body, that is, the fulfilment of condition (1).

Numerical calculations were carried out for the following values of the structural, kinematic and power parameters of this vibration dryer design:

- mass of the body $m=510 \mathrm{~kg}$;

- rotation frequency of debalances $n_{d}=2100$ $\mathrm{rpm} \cdot \mathrm{min}^{-1}$

- mass of the debalance $m_{d}=10 \mathrm{~kg}$;

- number of debalances $k_{d}=2$;

- diameter of the circle of rotation of the centre of mass of the debalance $d_{d}=0.01 \mathrm{~m}$;

- maximum stiffness of the support springs $C_{p}=8 \cdot 10^{5} \mathrm{~N} \cdot \mathrm{m}^{-1}$.

In addition, to build the necessary graphs, based on the results of the solution on a PC, we considered 
certain ranges of the corresponding parameters. Based on the numerical calculations, carried out on the PC, according to the compiled program of numerical calculations, we obtained a number of basic graphical dependencies, confirming the correct choice of the discussed constructive, kinematic and dynamic parameters of this vibration dryer, at which the necessary process of vibratory boiling of the food waste mixture inside the oscillating body and the satisfactory course of the drying process of the waste is ensured (Figure 3) - (Figure 7). Thus in Figure 3 there is shown dependence of acceleration $\ddot{x}$ of the vibration dryer body, obtained from the results of analytical calculations, on time $t$. Besides, the initial period of oscillations of the vibration dryer body is considered.

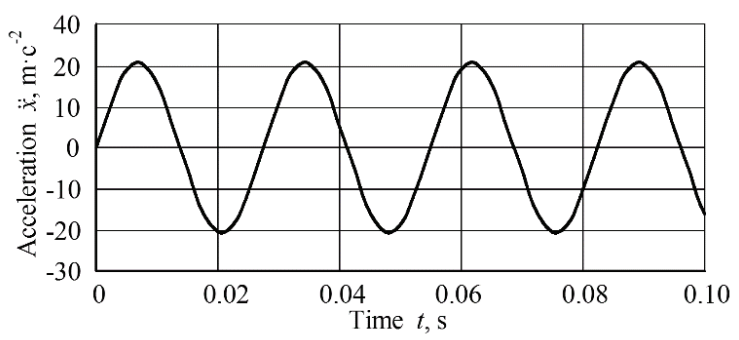

Figure 3. Dependence of acceleration of the vibration dryer body on time $t$.

As the graph, shown in Figure 3, with the above parameters, the change in the acceleration value of the vibration dryer body during its vertical oscillations occurs according to a sinusoidal law with amplitude of $20 \mathrm{~m} \cdot \mathrm{s}^{-2}$ (i.e, more than 2). Consequently, condition (1) of ensuring the process of vibrating boiling of the food waste mixture inside the housing is fulfilled. In addition, acceleration of the vibrational movement of the vibration dryer body changes both in magnitude and direction, which further contributes to the liquefaction of the indicated waste mixture. Figure 4 presents a similar dependence of acceleration of the vibration dryer body $\ddot{x}$ on the number of revolutions $n_{d}$ the debalance motor. Analysis of the graph, presented in Figure 4, witnesses that a rational frequency of rotation of the debalances of the vibration exciter is in the range of $2480 \ldots 2650 \mathrm{rpm} \cdot \mathrm{min}^{-1}$, at which the acceleration of the body $\ddot{x}$ reaches $27 \mathrm{~m} \cdot \mathrm{s}^{-2}$, which fully ensures the state of vibroboiling of the waste mixture inside the body. However, the value of the number of revolutions, equal to $1950 \ldots .2100 \mathrm{rpm} \cdot \mathrm{min}^{-1}$ of the debalance rotation also provides the required acceleration of the body $\ddot{x}$, which in this case is $18 \mathrm{~m} \cdot \mathrm{s}^{-2}$ and which is almost equal to 2 , ensuring the required quality of the waste drying.

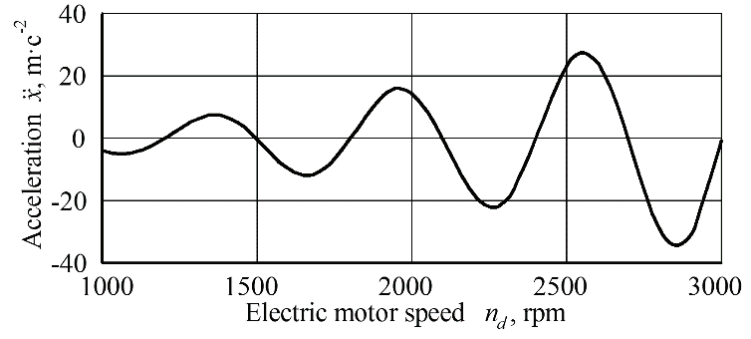

Figure 4. Dependence of acceleration of the vibration dryer body $\ddot{x}$ on the number of revolutions $n_{d}$ of the debalance.

Figure 5 shows a graph of the dependence of the acceleration $\ddot{x}$ of the vibration dryer body on the debalance mass $m_{d}$. From the graph, shown in Figure 5, it follows that the most rational range of the debalance mass $m_{d}$ values is a value equal to $10 \ldots 16$ $\mathrm{kg}$, at which the body acceleration $\ddot{x}$ varies from 20 to $30 \mathrm{~m} \cdot \mathrm{s}^{-2}$, that is, it is within the range from 2 to 3 , which fully satisfies condition (1).

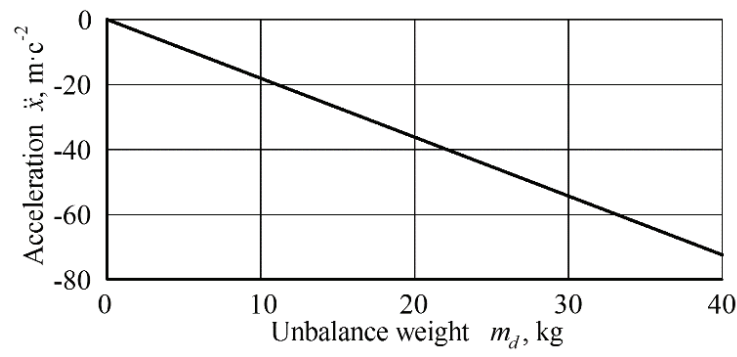

Figure 5. Dependence of acceleration of the vibration dryer body $\ddot{x}$ on the debalance mass $m_{d}$.

Dependence of acceleration $\ddot{x}$ of the vibration dryer body on the stiffness $C_{p}$ of the springs is shown in Figure 6.

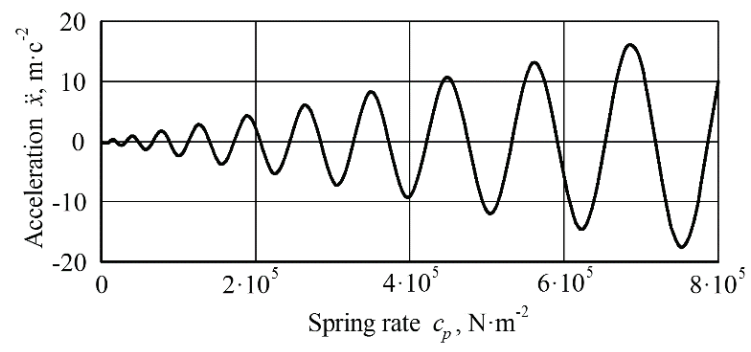

Figure 6. Dependence of acceleration of the vibration dryer body $\ddot{x}$ on the stiffness $C_{p}$ of the springs.

The graph, presented in Figure 6, shows that increasing the stiffness of the springs $C_{p}$, the acceleration of the body $\ddot{x}$ increases, which indicates an increase in the frequency of natural oscillations of the body of the vibration dryer and a decrease in the 
amplitude of its forced vibrations. Figure 7 presents a dependence graph of acceleration $\ddot{x}$ of the vibration dryer body on the mass $m$ of its body. Analysis of the graph, presented in Figure 7, shows that a change in the mass $m$ of the vibration dryer body within the range of $250 \ldots 500 \mathrm{~kg}$ causes a change in its acceleration in the range of $30 \ldots 20 \mathrm{~m} \cdot \mathrm{s}^{-2}$ (3...2, respectively), which ensures guaranteed vibroboiling of the waste mixture inside the vibration dryer body. So the parameters, obtained on the basis of the constructed mathematical model of the longitudinal vertical vibrations of the body of the vibration dryer, guarantee an efficient process of vibration drying of the waste obtained in the production of food products.

In a similar way, if necessary, you can solve equations (17) - (19). Next, we will define other parameters that characterise the vibration drying process of the waste obtained in the production of the food products. Thus the main parameter of the drying efficiency is the total heat consumption $Q$, determined according to (Anytipov, Kretov \& Ostrikov, 2001) using the following relationship:

$$
Q=Q_{s}+Q_{o}+Q_{\text {pot }},
$$

where $Q_{s}, Q_{o}, Q_{p o t}$ - correspondingly, the heat consumption for the actual drying process, for heating the solid phase of the waste and for losses in the environment. Besides, component $Q_{c}$ can be found by the formula:

$$
Q_{s}=\frac{\left(m_{o}-m_{c . o}\right)\left[\left(r_{p}+c_{p} T_{v}\right)-c_{v} t_{o 1}\right]}{\left[24 \cdot 3600-\left(t_{z}+t_{r}\right)\right]},
$$

where $r_{p}, c_{p}$ - respectively, the vaporisation heat and the heat capacity of steam; $T_{v}$ - the air temperature around the dryer; $c_{v}$ - the heat capacity of water; $t_{\mathrm{o} 1}-$ temperature of the waste at the entrance to the dryer.

Heat consumption for heating the solid phase:

$$
Q_{o}=\frac{m_{s . o} \cdot c_{s . o}\left(t_{o 2}-t_{o 1}\right)}{\left[24 \cdot 3600-\left(t_{z}+t_{r}\right)\right]},
$$

where $c_{\text {s.o }}$ - specific heat capacity of the dried waste; $t_{02}$ - their final temperature, approximately equal to the temperature $T_{T}$ of the heat carrier in the heating pipes.

The last component in formula (41) is found as:

$$
Q_{\text {pot }}=0.1 \cdot Q_{s} \text {. }
$$

Knowing $Q$, one can calculate the required heat exchange surface area $-S_{t . p . n}$ of the heating pipes:

$$
S_{t . p . n}=\frac{Q}{K_{p}\left(T_{T}-t_{o 1}\right)},
$$

where $K_{p}$ - the heat transfer coefficient (kinetic coefficient).

So, to ensure the pre-set drying capacity, the following condition must be met:

$$
S_{t . p} \geq S_{t . p . n}
$$

if this condition is not satisfied, parameters $n_{t}, r_{t}$ are selected according to the heat exchange surface area $S_{t . p . n}$ and the length $l_{t}$ of the heating pipes.

Also, by size $Q$, one can calculate the required mass $G_{t . t}$ of the heat carrier supplied through the heating pipes:

$$
G_{t . t}=\frac{Q}{c_{p}\left(T_{T}-t_{o 1}\right)},
$$

which can then be compared with the actual mass $G_{t}$ :

$$
G_{t}=n_{t} \cdot \pi \cdot r_{t}^{2} \cdot l_{t} \cdot \rho_{t} \geq G_{t . t},
$$

where $\rho_{t}$ - density of the heat carrier in the heating pipes.

When implementing the mode of continuous drying of the food waste, the required average speed $v_{o}$ of the movement of the waste in the body of the vibrating dryer, taking into account the pre-set daily efficiency of the proposed equipment, is found as:

$$
v_{o}=\frac{m_{o}}{24 \cdot 3600 \cdot \rho_{o . o} \cdot S_{p}},
$$

where $S_{p}$ - flow area of the screw feeder.

Then the required flow area $S_{z}$ of the gap between the shutters will be equal to:

$$
S_{z}=\frac{m_{o}}{\rho_{o . o} \cdot v_{o}} .
$$

Determination of the heat costs for the implementation of the working process, using a continuous drying mode, is carried out according to formulas (41) - (43). Let us calculate dependencies $S_{t . p . n}$ when $K_{p}$ and $t_{\mathrm{o} 2}$ change; and then, with a minimum fixed $S_{t . p . n}$ and $G_{t . t}$, let us calculate and build dependencies for the main design parameters of the pipes: $r_{t}, l_{t}, n_{t}$. For calculation we will use formulas (20) - (23), as well as (41) - (43) and the following initial parameters: $\mathrm{T}_{s}=10000 \mathrm{~kg} ; r_{s}=0.4 \mathrm{~m}$; $D_{s}=0.8 \mathrm{~m} ; d_{s}=0.7 \mathrm{~m} ; n_{s}=0.01 \mathrm{~s}^{-1} ; t_{s}=0.25 \mathrm{~m}$; $\rho_{o . o}=950 \mathrm{~kg} \cdot \mathrm{m}^{-3} ; \rho_{s . o}=162 \mathrm{~kg} \cdot \mathrm{m}^{-3} ; \mathrm{T}_{s}=1 ; k_{s}=2$; $b_{1}^{o . o}=0.02 \mathrm{~m} ; b_{2}=0.025 \mathrm{~m} ; \alpha=6.3^{\mathrm{o}} ; k_{1}=0.95$; $k_{2}=0.52 ; k_{3}=0.9 ; r_{p}=2258 \mathrm{~kJ} \cdot \mathrm{g}^{-1} ; c_{p}=2135 \mathrm{~J} \cdot \mathrm{kg}^{-1}$ $\cdot \mathrm{K}^{-1} ; c_{v}=1877 \mathrm{~J} \cdot \mathrm{kg}^{-1} \cdot \mathrm{K}^{-1} ; t_{\mathrm{o} 1}=20^{\circ} \mathrm{C} ; T_{v}=20{ }^{\circ} \mathrm{C}$; $c_{\text {s.o }}=1100 \mathrm{~J} \cdot \mathrm{kg}^{-1} \cdot \mathrm{K}^{-1} ; v_{k}=1 \mathrm{~m} \cdot \mathrm{s}^{-1} ; B_{k}=1.5 \mathrm{~m} ; h_{k}=$ $0.1 \mathrm{~m} ; t_{\mathrm{o} 2}=50 \ldots 100{ }^{\circ} \mathrm{C} ; K_{p}=150 \ldots 2500 ; \Pi_{t}=50$ $\ldots 200 ; l_{t}=1 \ldots 5 \mathrm{~m} ; r_{t} \stackrel{p}{=} 0.025 \ldots 0.1 \mathrm{~m}$. In order 


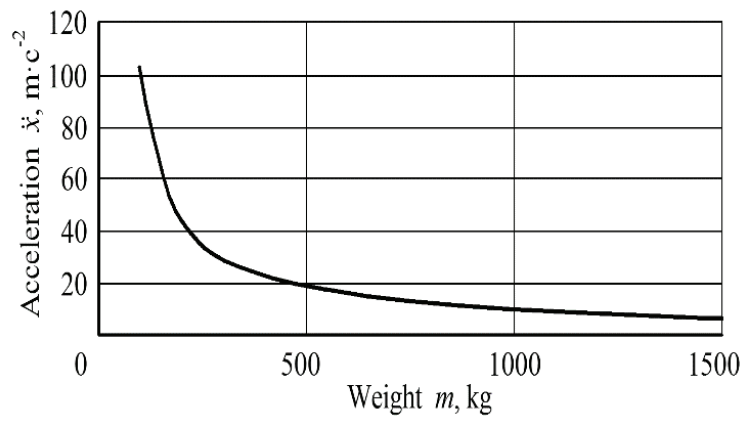

Figure 7. Dependence of acceleration of the vibration dryer body $\ddot{x}$ on the mass $m$ of its body.

to perform numerical calculations on a PC Microsoft Excel 2016, we compiled a program with the help of which we obtained and built graphical dependencies of the technological parameters that ensure efficient dehydration of the mass of food waste from the design parameters of the vibration dryer, shown in (Figure 8$)-($ Figure 12). So, in Figure 8 there is shown dependence of the heat transfer area $S_{t . p . n}$ upon temperature $t_{\mathrm{o} 2}$. For this case we have chosen the maximum value $K_{p}=2500$.

Analysis of the graphical dependence, presented in Figure 8, indicates that, in order to transfer a greater mass of the waste with a greater amount of heart, which is achieved by heating to a higher temperature $t_{\mathrm{o} 2}$, a large heat exchange surface area $S_{t . p . n}$ is required. In addition, an area of $S_{t . p . n}=4.15 \mathrm{~m}^{2}$ is required to ensure the optimum temperature $t_{02}=$ $100{ }^{\circ} \mathrm{C}$. It should also be noted that the obtained dependence $S_{t . p . n}=f\left(t_{\mathrm{o} 2}\right)$ is of a linear nature. Figure 9 presents dependence of the heat transfer area $S_{t . p . n}$ upon the heat transfer coefficient $K_{p}$. At the same time, we considered a case when $t_{\mathrm{o} 2}=100^{\circ} \mathrm{C}$. Based on the graphical dependence, presented in Fig. 9, area $S_{t . p . n}$ and, together with it, the dimensions of

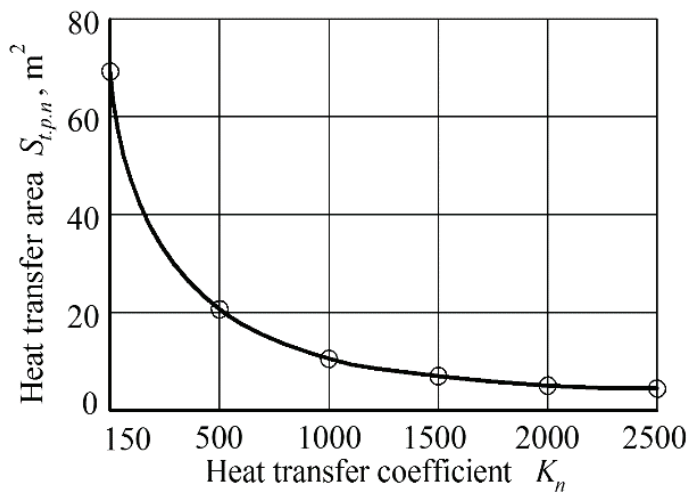

Figure 9. Dependence of the heat transfer area $S_{t . p . n}$ upon the heat transfer coefficient $K_{p}$ at $\mathrm{t}_{\mathrm{o} 2}=100{ }^{\circ} \mathrm{C}$.

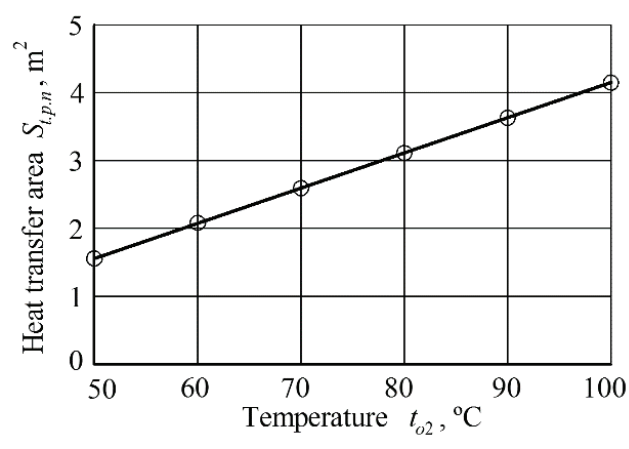

Figure 8. Dependence of the heat transfer area $S_{\text {t.p.n }}$ upon temperature $\mathrm{t}_{\mathrm{o} 2}$ at $K_{p}=2500$.

the vibration dryer body significantly depend on the type of heat carrier passing through the heating pipes and on the heat transfer coefficient $K_{p}$ that it ensures. The highest efficiency of the vibration dryer of the proposed design and its working process is achieved in the case of using condensed water vapour as a heat carrier, for which $K_{p}=2500$ (Mohseni et al., 2017) however, already at $K_{p}=1500$ the required area $S_{t . p . n}$ is close to the minimum.

Dependence of the length $l_{t}$ of the pipe on radius $r_{t}$ of the pipe is indicated in Figure 10. According to the graph, shown in Figure 10, at a constant number of pipes $n_{t}=100$, which can be considered the maximum permissible by their dimensions, and with an increase in the radius $r_{t}$ of the pipes from 0.025 to $0.075 \mathrm{~m}$ (a range that includes the most common pipe radii for the food industry), their required length $l_{t}$ significantly decreases. Besides, values $l_{t}=5 \ldots 2 \mathrm{~m}$, acceptable for most food industry enterprises, are provided at $r_{t} \approx 0.05 \ldots 0.08 \mathrm{~m}$. Figure 11 shows dependence of the pipe radius $r_{t}$ on their number $n_{t}$.

Taking into account the dependence, presented in Figure 11, with an unchanged maximum permissible value for most cases of $l_{t}=5 \mathrm{~m}$ and an increase in

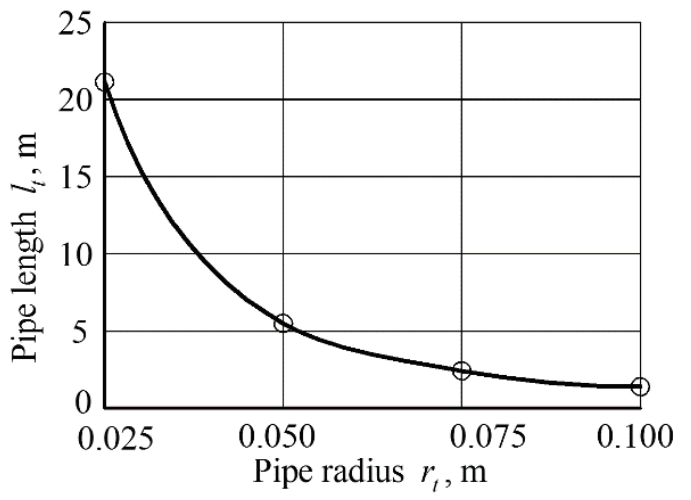

Figure 10. Dependence of the length of the pipe $l_{t}$ on the radius of the pipe $r_{t}$ at: $\mathrm{t}_{\mathrm{o} 2}=100{ }^{\circ} \mathrm{C}$;

$$
n_{t}=100 ; K_{p}=2500 \text {. }
$$




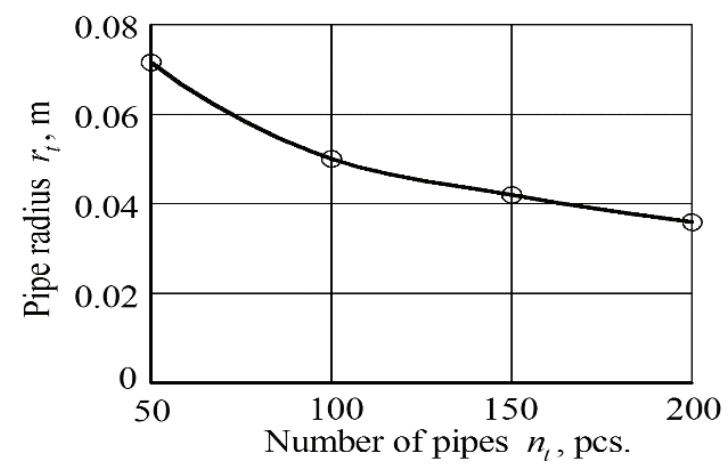

Figure 11. Dependence of the pipe radius $r_{t}$ on the number of pipes $n_{t}: l_{t}=5 \mathrm{~m} ; K_{p}=2500 ; \mathrm{t}_{\mathrm{o} 2}=100{ }^{\circ} \mathrm{C}$.

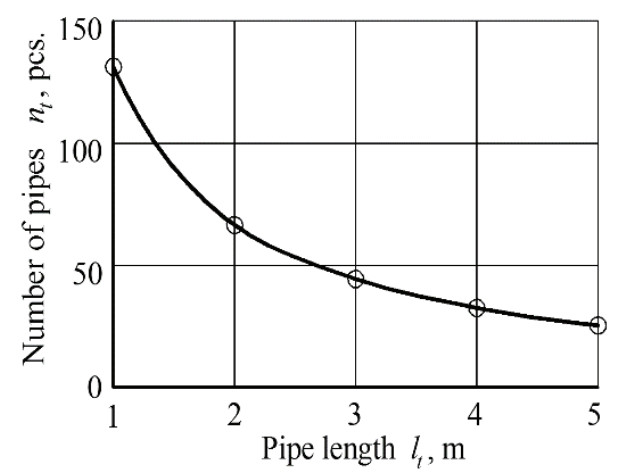

Figure 12. Dependence of the number of pipes $n_{t}$ upon the length of the pipe $l_{t}: r_{t}=0.1 \mathrm{~m}$; $K_{p}=2500 ; \mathrm{t}_{\mathrm{o} 2}=100^{\circ} \mathrm{C}$. the number of pipes $n_{t}$ from 50 to 200 (the latter value is the limit), the radius of the pipes $r_{t}$ is within the acceptable range of lengths: $r_{t}=0.072 \ldots 0.036 \mathrm{~m}$. From the graphs, shown in Figure 10 and Figure 11, it is also obvious that, when the basic design parameters of pipes within the permissible limits are changed, $r_{t}$ depends more on $l_{t}$ than on $n_{t}$. And, finally, in the case of a constant pipe radius $r_{t}=0.1 \mathrm{~m}$, with an increase of $l_{t}$ from 1 to $5 \mathrm{~m}$, we obtained a graphical dependence (Figure 12), according to which $n_{t}$ decreases from 132 to 26 , i.e. within permissible limits, which allows the selection of the main design parameters of the pipes and, together with them, the dimensions of the dryer body, taking into account the conditions and requirements of the corresponding efficiency of the dryer.

As it follows from the graphical dependence, presented in Figure 12, the most rational design parameters of the considered vibration dryer in order to achieve the pre-set efficiency of the working process and the final moisture content of the dewatered waste are: $r_{t}=0.1 \mathrm{~m} ; l_{t}=3 \mathrm{~m} ; n_{t}=50$, at $K_{p}=2500$ and $t_{\mathrm{o} 2}=100{ }^{\circ} \mathrm{C}$. In such a way all the main structural, kinematic, dynamic and technological parameters of the vibratory dryer have been obtained, which ensure efficient dehydration of the waste obtained in the production of food products.

\section{Conclusions}

1. A new design of the dryer has been developed, which creates conditions for efficient drying of the waste obtained in the production of food products, in which the conditions of vibro-boiling conditions are ensured when the body is subject to vertical oscillations. A mathematical model of the considered vibration dryer has been built. On the basis of the developed equivalent scheme differential equations of the translational vertical oscillations of its body along with the processed waste are obtained.
2. It has been determined that the rational parameters of a vibration dryer, providing vibroboiling of the mass of food waste, can be obtained with a body mass of $m=250 \ldots 510 \mathrm{~kg}$; a mass of the debalances $m_{d}=10 \ldots 15 \mathrm{~kg}$; the number of revolutions of the unbalance electric motor $n_{d}=$ $1950 \ldots 2650 \mathrm{rpm} \cdot \mathrm{min}^{-1}$; maximum rigidity of the support springs $C_{p}=8 \cdot 10^{5} \mathrm{~N} \cdot \mathrm{m}^{-1}$; the diameter of rotation of the centre of the mass of debalances $d_{d}=0.01 \mathrm{~m}$.

3. As a result of the conducted thermophysical theoretical and experimental studies of the vibration drying process and calculations on the $\mathrm{PC}$, rational values of the heat transfer area were found $S_{t . p . n}=4.15 \mathrm{~m}^{2}$; the radius of the heating pipe $r_{t}=0.1 \mathrm{~m}$; the length of the heating pipe $l_{t}=3 \mathrm{~m}$; the number of the heating pipes $n_{t}=50$; the heat transfer coefficient $K_{p}=2500$; the final temperature of the dried waste $t_{o 2}=100{ }^{\circ} \mathrm{C}$.

\section{References}

Anytipov, S., Kretov, I. \& Ostrikov, A. (2001). Machines and apparatus for food production. Moscow, 703 p.

Bulgakov, V., Holovach, I., Bandura, V. \& Ivanovs, S. (2017). A theoretical research of the grain milling technological process for roller mills with two degrees of freedom. INMATEH Agricultural Engineering, 52(2), pp. 99-1Formas sākumsFormas beigas06. DOI: https://inmateh. eu/volumes/old-volume/volume-52-no-2-2017/ article/a-theoretical-research-of-the-grainmilling-technological-process-for-roller-millswith-two-degrees-of-freedom

Dreizler, R.M. \& Ludde C.S. (2010). Theoretical Mechanics. Springer, $402 \mathrm{p}$.

Handayani, S., Utomo, T. \& Yulianto, M. (2017). Performance evaluation of continuous vibrating fluidized bed dryer on green tea production. 
Advanced science letters, 23. pp. 2530-2532. DOI: 10.1166/asl.2017.8676.

Iskovich-Lotocky, R., Obertuh, R. \& Sevostianov, I. (2006). Processes and machines of vibration and vibration impact technologies. Vinnica, Ukraine, $291 \mathrm{p}$.

Jakovlev, S., Volkov, L., Voronov, Y., \& Volkov, V. (1999). Treatment and disposal of industrial waste water sludge. Moscow, $448 \mathrm{p}$.

Lehmann, S.E., Hartge, E.-U., Jongsma, A., Leeuw, I.M., Innings, F. \& Heinrich, S. (2019). Fluidization characteristics of cohesive powders in vibrated fluidized bed drying at low vibration frequencies. Powder Technology, Vol. 357, pp.54-63, DOI: 10.1016/j.powtec.2019.08.105.

Mohseni, M., Kolomijtschuk, A., Bernhard, P. \& Demoulling, M. (2019). Biomass drying in a vibrating fluidized bed dryer with a LagrangianEulerian approach, International Journal of Thermal Sciences, Volume 138, 2019, pp. 219-234, ISSN 1290-0729, DOI: 10.1016/j. ijthermalsci.2018.12.038.

Ostrikov, A., Krasovicki, Y. \& Petrov, S. (2006). Processes and apparatus of food production. Saint Petersburg, 559 p.

Ovchinnikov, N. \& Natareev, S. (2009). Drying and firing in a fluidized bed. Ivanovo, Russia, $106 \mathrm{p}$.
Sazhin, B. (1984). Foundations of the theory of drying. Moscow, Russia, 320 p.

Sevostianov, I. (2020). Technology and equipment for vibroimpact dehydration of dispersed materials. Vinnica, Ukraine, 303 p.

Sevostianov, I. \& Lucik, V. (2017). Installation for multistage dehydration of food waste. Bulletin of mechanical engineering and transport, No 1, pp.105 - 113

Sevostianov, I., Poliscuk, O. \& Slabky, A. (2015). Development and research of an installation for two-component vibro-shock dehydration of food waste. Eastern European Journal of Advanced Technologies, No.7 (77), pp. 40 - 46.

Yogendrasasidhar, D. \& Setty Pydi Y. (2019). Experimental studies and thin layer modelling of pearl millet using continuous multistage fluidized bed dryer staged externally, Engineering Science and Technology International Journal, Volume 22, Issue 2, pp. 428-438. DOI: https://doi. org/10.1016/j.jestch.2018.10.010

Wang, X., Zhang, Z., \& Deng, Y. (2011). The harmonic response analysis on vibration system of disc vibration dryer. Applied mechanics and materials, 84-85, pp.209-213. DOI: 10.4028/ www.scientific.net/AMM.84-85.209. 